\section{Protective role of $\mathrm{MeCP} 2$ in SSc}

Methyl-CpG-binding protein 2 (MeCP2) is dysregulated in fibroblasts from patients with systemic sclerosis (SSc) and might have a protective role against fibrosis, according to results from a new study. $\mathrm{MeCP} 2$ is an epigenetic regulator that binds to methylated DNA.

SSc is a rare disease characterized by autoimmune damage, vascular dysfunction and fibrosis. "We have been using epigenetic characterization on disease-relevant cell types to try to better understand SSc so that better treatment options can be developed," explains corresponding author Amr Sawalha. "We focused this study on $\mathrm{MeCP} 2$ as our group was the first to describe a genetic association between genetic variants in MeCP2 and autoimmunity over a decade ago."
The researchers found increased expression of MeCP2 in fibroblasts from patients with diffuse cutaneous $\mathrm{SSc}$ (dcSSc) compared with fibroblasts from healthy individuals.

Next, the researchers overexpressed MeCP2 in normal dermal fibroblasts. Interestingly, this overexpression of MeCP2 suppressed myofibroblast differentiation and fibroblast proliferation and migration.

RNA sequencing was subsequently used to identify downstream target genes regulated by $\mathrm{MeCP} 2$ in dcSSc fibroblasts with and without $M e C P 2$ knockout. Three of the genes identified were PLAU, NID2 and ADA. Functional studies demonstrated that the products of these genes mediated the antifibrotic effects of MeCP2.

\section{Fibrosis}

\title{
Location, location, location
}

The T cell receptor $\beta$ chain (TCR $\beta$ ) repertoire in the synovial tissue of patients with rheumatoid arthritis (RA) has a high degree of similarity between different joints and between different locations in the same joint, according to new research.

Previous studies have produced conflicting results as to whether the same $T$ cell clones drive inflammation in different joints. To address this question, the authors of the present study used next generation sequencing to analyse the entire TCR $\beta$ repertoire of T cells from synovial tissue, synovial fluid and peripheral blood of patients with RA.

"With the advent of novel sequencing technologies, we were able to study the full repertoire of TCR $\beta$ clones quantitatively and with unprecedented detail and accuracy in a larger group of patients than had previously been possible, comparing synovial tissues within and between joints," says corresponding author Niek de Vries. "The results indicate that shared $\mathrm{T}$ cell responses are indeed responsible for the inflammation in different joints."

The researchers compared the overlap between the top 25 most expanded TCR $\beta$ clones at different sites in the same joint and in different inflamed joints in the same patient, and found an overlap of $\sim 50 \%$ in both instances. This high degree of similarity suggests that inflammation is driven by a limited number of clones. "These observations are good news since they support the feasibility of immunotherapies that selectively target dominant $\mathrm{T}$ cell responses," states de Vries.

Interestingly, the TCR $\beta$ repertoires of synovial tissue and synovial fluid from the same joint shared only a low degree of similarity. "This result indicates that for the study of lymphocyte specificity, synovial tissue and synovial fluid are not interchangeable," explains de Vries.
Credit: Susanne Harris/Macmillan Publishers Limited

...increased
MeCP2
expression
in dcSSc
fibroblasts
might be
a defence
mechanism...

"We showed that manipulating these genes can mitigate several of the pathologic features in SSc fibroblasts," says Sawalha.

The authors suggest that increased MeCP2 expression in dcSSc fibroblasts might be a defence mechanism against fibrosis. "Manipulating MeCP2 or molecules encoded by the MeCP2-target genes we identified could be of therapeutic potential in this debilitating disease," concludes Sawalha. Future studies of lung fibroblasts or other cell types involved in SSc might also be warranted.

Isobel Leake

ORIGINAL ARTICLE He, Y.et al. Methyl-CpGbinding protein 2 mediates antifibrotic effects in scleroderma fibroblasts. Ann. Rheum. Dis. https:// doi.org/10.1136/annrheumdis-2018-213022 (2018)
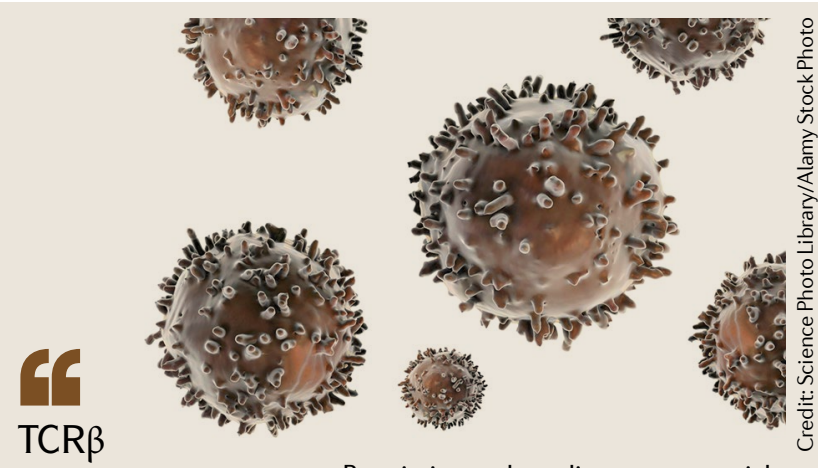

repertoires of synovial tissue and synovial fluid from the same joint shared only a low degree of similarity

Restricting such studies to use synovial tissue alone will inevitably increase demand for a scarce resource that is normally collected by arthroscopy-guided biopsy. However, the high degree of similarity in the TCR $\beta$ repertoire at different synovial tissue locations in the same joint suggests that synovial tissue could potentially be gathered using a less targeted and less time-consuming technique, thereby increasing the supply of tissue for research.

Joanna Collison doi.org/10.4049/jimmunol.1800421 (2018) 